\title{
Das Flaviussche Sieb
}

\author{
von \\ Mats ErIK Andersson (Stockholm)
}

Problemstellung. Es sei $\mathcal{F}_{1}=\{1,2,3, \ldots\}$ die Menge der natürlichen Zahlen. Diese Menge wird gesiebt, indem jedes zweite Element entfernt wird und die ungeraden Zahlen übrigbleiben. Der Rest wird mit $\mathcal{F}_{2}$ bezeichnet. Danach wird jedes dritte Element in $\mathcal{F}_{2}$ weggenommen; so $\mathcal{F}_{3}=$ $\{1,3,7,9, \ldots\}$. Die Menge $\mathcal{F}_{k}$ entsteht indem man aus der Menge $\mathcal{F}_{k-1}$ jedes $k$-te Element streicht. Man schreibe nun $\mathcal{F}_{\infty}=\bigcap_{k=1}^{\infty} \mathcal{F}_{k}$ und suche die Anzahlfunktion $F(n)=\left|\mathcal{F}_{\infty} \cap[1, n]\right|$ zu bestimmen.

Diese Fragestellung scheint zum ersten Mal in Nordisk Matematisk Tidskrift [1] erwähnt worden zu sein und dort hat Viggo Brun eine Lösung angegeben. Der Name „das Flaviussche Sieb“ stammt von Gardiner u.a. [5], allerdings ohne jede Begründung. Doch scheint eben dieser „Flavius“ mit einem Ereignis bei Armageddon verknüpft zu sein. Die Lösungen in [1] geben $F(n) \sim \frac{2}{\sqrt{\pi}} \sqrt{n}$ an. Eine Präzisierung stammt von Brun [3]:

$$
F(n)=\frac{2}{\sqrt{\pi}} \sqrt{n}+\theta(n) n^{3 / 8}, \quad|\theta(n)|<1,13, n>5^{8} .
$$

In dieser Arbeit werden wir zeigen:

SATZ.

$$
F(n)=\frac{2}{\sqrt{\pi}} \sqrt{n}+\mathcal{O}\left(n^{1 / 6}\right)
$$

1991 Mathematics Subject Classification: 11B83, 11N35.

Diese Arbeit ist ursprünglich während eines längeren Aufenthaltes in Stuttgart entstanden. Sie diente als eine Art Abschlußarbeit für mein Grundstudium. Die Anregung zum Problemkreis hat der vorzeitig verstorbene Dr. rer. nat. Gerold Wagner geleistet. Seinem Andenken ist diese Veröffentlichung gewidmet. Er ist im März 1990 bei einem Lawinenunfall ums Leben gekommen. In der kurzen Zeit unserer Freundschaft hat er mich vielfältig beeinflußt, sowohl privat als auch wissenschaftlich. 
Einleitung, Hilfssätze

Hilfssatz 1. Es sei

$$
B(n)=\frac{(2 n) ! !}{(2 n+1) ! !}=\frac{2^{2 n} n !^{2}}{(2 n+1) !} .
$$

Dann gilt:

(1) $B(n)$ ist streng monoton fallend, und $\sum_{k=1}^{n} B(k)=2(n+1) B(n)-2$.

(2) $B(n)=\frac{\sqrt{\pi}}{2 \sqrt{n+1}}\left(1+\frac{1}{8(n+1)}+\mathcal{O}\left(n^{-2}\right)\right)$.

Bemerkung. Einen schönen Beweis von der Summe in (1) hat Carlitz [4] gefunden.

Es bezeichne fortan $[x]$ die Gauss'sche Klammer, d.h. die größte ganze Zahl kleiner oder gleich $x$. Mehrmals wird aus $\left[\frac{a}{b}\right]=c$, wobei $a$ und $b$ ganzzahlig sind, die Folgerung $b c \leq a \leq b c+(b-1)$ angewandt.

Definition. Zu den Mengen $\left\{\mathcal{F}_{k}\right\}_{k=1}^{\infty}$ und zu einem festen $n$ führen wir drei Größen ein, deren Studium zur Entwicklung des Siebverfahrens führt: $N_{k}=\left|\mathcal{F}_{k} \cap[1, n]\right|, m_{k}=N_{k-1}-N_{k}$ und $h_{k}=m_{k}-m_{k+1}$. Ihre Abhängigkeit von $n$ wird unterdrückt. Da im $k$-ten Schritt jedes $k$-te Element in $\mathcal{F}_{k-1} \cap[1, n]$ unter den $N_{k-1}$ möglichen Zahlen entfernt wird, ist $m_{k}=$ $\left[N_{k-1} / k\right]$ einleuchtend.

Beim Fortschreiten des Verfahrens sind zwei „Zeitpunkte“ interessant: $k_{0}$ sei der größte Index mit $m_{k}>0$ und es bezeichne $k^{*}=\max \left\{k \mid h_{k} \geq 2\right\}$. Insbesondere gilt für $k \in\left\{k^{*}+1, \ldots, k_{0}\right\}$ entweder $m_{k+1}=m_{k}-1$ oder $m_{k+1}=m_{k}$. Außerdem ist $F(n)=\lim _{k \rightarrow \infty}\left|\mathcal{F}_{k} \cap[1, n]\right|=N_{k_{0}}$.

Hilfssatz 2.

$$
\begin{gathered}
(k-1) N_{k-1} \leq k N_{k} \leq(k-1) N_{k-1}+(k-1), \\
n \leq k N_{k} \leq n+\frac{1}{2} k(k-1), \\
k_{0}-1 \leq N_{k_{0}}=F(n) \leq k_{0} \\
\sqrt{n}-\frac{1}{2}<F(n)<\sqrt{2 n} .
\end{gathered}
$$

B ew eis. Die Ungleichung (1) folgt aus $N_{k}=N_{k-1}-m_{k}=N_{k-1}-$ $\left[N_{k-1} / k\right]$ durch Auflösen der Klammer. Das Wiederholen von (1) liefert (2).

Da nach Konstruktion $N_{k-1} \geq N_{k}$ gilt, ist offenbar $N_{k-1} / k$ mit wachsendem $k$ streng abnehmend. Folglich ist $m_{k}=\left[N_{k-1} / k\right]$ nicht wachsend. Dadurch ist auch $k_{0}$ mittels $m_{k_{0}} \geq 1$ und $m_{k_{0}+1}=0$ eindeutig bestimmt. Die zwei Bedingungen besagen $\left[N_{k_{0}-1} / k_{0}\right] \geq 1$ und $\left[N_{k_{0}} /\left(k_{0}+1\right)\right]=0$, d.h. $N_{k_{0}-1} \geq k_{0}$ bzw. $N_{k_{0}}<k_{0}+1$. Wegen der Ganzzahligkeit ergibt sich $N_{k_{0}} \leq k_{0}$. 
Es ist weiter einleuchtend, daß $k_{0}=k_{0}(n)$ mit wachsendem $n$ niemals abnehmen kann. Insbesondere ist für $n \geq 5$ immer $k_{0} \geq 3$. Für jene $n$ gilt wegen (1) die Beziehung $N_{k_{0}-1} / k_{0} \leq N_{k_{0}} /\left(k_{0}-1\right) \leq k_{0} /\left(k_{0}-1\right) \leq 3 / 2$, d.h. $m_{k_{0}}=1$. Damit haben wir $k_{0}-1 \leq N_{k_{0}-1}-1=N_{k_{0}-1}-m_{k_{0}}=N_{k_{0}}$ allgemein bestätigt und (3) ist erledigt für $n \geq 5$. Nachrechnen mit $n=2$, 3 und 4 belegt (3) auch in diesen Fällen.

Aus (2) mit $k=k_{0}$ samt (3) folgern wir

$$
n \leq k_{0} N_{k_{0}} \leq N_{k_{0}}\left(N_{k_{0}}+1\right)<\left(N_{k_{0}}+\frac{1}{2}\right)^{2} .
$$

Anderseits gilt $k_{0} N_{k_{0}} \leq n+\frac{1}{2} k_{0}\left(k_{0}-1\right) \leq n+\frac{1}{2} k_{0} N_{k_{0}}$, d.h. $k_{0} N_{k_{0}} \leq 2 n$, beziehungsweise $N_{k_{0}}^{2} \leq k_{0} N_{k_{0}}^{2}$. Da beim Einsetzen von (3) in der Form $k_{0}-1 \leq N_{k_{0}}$ bzw. $N_{k_{0}} \leq k_{0}$ nicht gleichzeitig Gleichheit in den beiden Beziehungen bestehen kann, folgern wir aus deren Kombination $N_{k_{0}}^{2}<2 n$.

Wir haben noch einige Beziehungen nötig, die wichtige Größenverhältnisse verdeutlichen. Diese sind kaum mehr als zurechtgelegte Formen des eben angeführten Hilfssatzes, für deren späteren Einsatz angepasst.

HiLfsSATZ 3. Für alle $k \geq 2$ gelten die Ungleichungen

$$
\begin{gathered}
m_{k}(k-1) \leq N_{k} \leq\left(m_{k}+1\right)(k-1), \\
\left(m_{k}-h_{k}\right)(k+1) \leq N_{k} \leq\left(m_{k}-h_{k}\right)(k+1)+k, \\
k(k-1)\left(m_{k}-\frac{1}{2}\right) \leq n \leq k(k-1)\left(m_{k}+1\right) .
\end{gathered}
$$

B ew eis. Gemäß Definition bestehen $m_{k}=\left[N_{k-1} / k\right]=\left[\left(m_{k}+N_{k}\right) / k\right]$ und $m_{k}-h_{k}=m_{k+1}=\left[N_{k} /(k+1)\right]$, woraus offensichtlich (5) bzw. (6) durch Auflösen der Klammer hervorgehen.

$\mathrm{Um}(7)$ zu beweisen, schreibt man zuerst $k N_{k}=m_{k} k(k-1)+k\left\{N_{k}-\right.$ $\left.m_{k}(k-1)\right\}$. Vermöge $(5)$ sieht man $N_{k}-m_{k}(k-1) \leq k-1$ ein. Hieraus folgt nun

$$
k N_{k} \leq m_{k} k(k-1)+k(k-1) .
$$

Anderseits ist nach (2)

$$
n \geq k N_{k}-\frac{1}{2} k(k-1) \geq m_{k} k(k-1)-\frac{1}{2} k(k-1)=\left(m_{k}-\frac{1}{2}\right) k(k-1),
$$

wobei in der letzten Ungleichung die Beziehung $N_{k} \geq m_{k}(k-1)$ aus (5) eingesetzt wurde. Der Hilfssatz ist bewiesen.

Die zulässigen Kombinationen von (5) und (6) geben

$$
\begin{aligned}
& m_{k}(k-1) \leq\left(m_{k}-h_{k}\right)(k+1)+k \quad \text { bzw. } \\
& \left(m_{k}-h_{k}\right)(k+1) \leq\left(m_{k}+1\right)(k-1) .
\end{aligned}
$$

Löst man in jeder Ungleichung die Zahl $k$ aus, ergibt sich sofort

$$
\frac{2 m_{k}-h_{k}+1}{h_{k}+1} \leq k \leq \frac{2 m_{k}-h_{k}}{h_{k}-1} \text {. }
$$


Die erste Beziehung in (8) führt auf $k \leq \ldots$ und die zweite auf $k \geq \ldots$ zu. Infolgedessen finden wir wiederum dank (6), daß

$$
N_{k} \leq k\left(m_{k}-h_{k}+1\right)+m_{k}-h_{k} \leq \frac{2 m_{k}-h_{k}}{h_{k}-1}\left(m_{k}-h_{k}+1\right)+m_{k}-h_{k} .
$$

Eine einfache Rechnung liefert schließlich

$$
N_{k} \leq m_{k}\left(2 m_{k}-2 h_{k}+1\right) /\left(h_{k}-1\right) .
$$

Die besondere Wahl $k=k^{*}, M=m_{k^{*}+1}=m_{k^{*}}-h_{k^{*}}$ führt zu (man beachte $h_{k^{*}} \geq 2$ )

$$
k^{*} \leq 2 M+2, \quad N_{k^{*}} \leq(M+2)(2 M+1) .
$$

Damit ergibt sich $n \leq k^{*} N_{k^{*}} \leq(2 M+2)(2 M+1)(M+2)<4\left(M+\frac{7}{6}\right)^{3}$ und wir haben den folgenden Satz bewiesen.

SATZ 4. Es gilt die Ungleichung

$$
m_{k^{*}+1}>\left(\frac{n}{4}\right)^{1 / 3}-\frac{7}{6}
$$

Die Filteridentität und ihre Folgerungen. Die Effektivität der hiesigen Methode basiert auf dem Zusammenführen aller Schritte, deren entsprechenden $h_{k}$ denselben Wert annehmen. Die so entstehende Beziehung nennen wir die Filteridentität:

SATZ 5. Es sei $n \geq 5$. Für $2 \leq k \leq k_{0}$ besteht die nachstehende Identität:

$$
\left(2 m_{k}-h_{k}\right)\left(k_{0}-k\right)=\left(N_{k}-k m_{k}\right)+\left(k_{0}-N_{k_{0}}\right)+\left(m_{k}-1\right) k_{0}+\sum_{l=k+1}^{k_{0}-1} h_{l}\left(k_{0}-l\right) .
$$

B ew e is. Gemäß Definition gilt $N_{l-1}-N_{l}=m_{l}\{l-(l-1)\}$, so daß eine Summation von $k+1$ nach $\kappa$ gibt

$$
N_{k}-N_{\kappa}=\sum_{l=k+1}^{\kappa} l m_{l}-\sum_{l=k}^{\kappa-1} l m_{l+1}=\kappa m_{\kappa+1}-k m_{k+1}+\sum_{l=k+1}^{\kappa} l h_{l} .
$$

Diese Identität kann man so umschreiben:

$$
\begin{aligned}
\left(m_{k}+m_{k+1}\right)(\kappa-k)= & \left(N_{k}-k m_{k}\right)+\left(\kappa m_{\kappa}-N_{\kappa}\right) \\
& +\left(m_{k}+m_{k+1}-m_{\kappa}-m_{\kappa+1}\right) \kappa-\sum_{l=k+1}^{\kappa} l h_{l} .
\end{aligned}
$$

$\operatorname{Dank} \sum_{l=k+1}^{\kappa} h_{l}=\sum_{l=k+1}^{\kappa}\left(m_{l}-m_{l+1}\right)=m_{k+1}-m_{\kappa+1}$ finden wir

$$
\left(2 m_{k}-h_{k}\right)(\kappa-k)=\left(N_{k}-k m_{k}\right)+\left(\kappa m_{\kappa}-N_{\kappa}\right)+\left(m_{k}-m_{\kappa}\right) \kappa+\sum_{l=k+1}^{\kappa-1} h_{l}(\kappa-l) \text {, }
$$


wobei auch $m_{k}+m_{k+1}=2 m_{k}-h_{k}$ angewandt wurde. Mit $\kappa=k_{0}$ und $m_{k_{0}}=1$ (wegen $n \geq 5$, vgl. den Beweis von Hilfssatz 2) folgt die Behauptung.

SATZ 6. Für alle $k$ mit $h_{k}=1$ und $k^{*}<k \leq k_{0}$ gilt die Abschätzung

$$
\left|k-B\left(m_{k}-1\right) k_{0}\right|<1 \text {. }
$$

B e w e is. Wir werden eine Induktion durchführen, die auf der nachstehenden Umformung basiert:

$$
\left|\left(k_{0}-l\right)-\left\{1-B\left(m_{l}-1\right)\right\} k_{0}\right|<1 \quad \text { wenn } \quad h_{l}>0, k^{*}<l \leq k_{0} .
$$

Diese Aussage ist trivial im Falle $l=k_{0}$, da die linke Seite gleich Null ist. Die Annahme $k^{*}<l$ führt dazu, daß $h_{l}=0$ oder 1 ist. Folglich ist jedes $m=1, \ldots, m_{l}$ unter den Zahlen $m_{l}, \ldots, m_{k_{0}}$ zu finden, sobald $k^{*}<l \leq k_{0}$. Außerdem stehen die Zahlen $j$ mit $l \leq j \leq k_{0}$ und $h_{j}=1$ durch $j \mapsto m_{j}$ in ein-ein-deutigem Verhältnis zu der Menge $\left\{1, \ldots, m_{l}\right\}$, wenn nur $h_{l}=1$ gilt.

Es sei nun (10) gültig für diejenige $l=k+1, \ldots, k_{0}$, die $h_{l}=1$ erfüllen, dabei darf ohne Einschränkung $h_{k}=1$ angenommen werden. Der Kürze halber sei im Folgenden $R_{k}=\left\{N_{k}-k m_{k}\right\}+k_{0}-N_{k_{0}}$ gesetzt. Die Filteridentität (Satz 5) und die Induktionsannahme beweisen nun

$$
\begin{aligned}
\left(2 m_{k}-1\right)\left(k_{0}-k\right)= & R_{k}+\left(m_{k}-1\right) k_{0}+\sum_{l=k+1}^{k_{0}-1} h_{l}\left(k_{0}-l\right) \\
< & R_{k}+\left(m_{k}-2\right)+\left(2 m_{k}-3\right) k_{0}-\sum_{l=k+1}^{k_{0}-1} h_{l} B\left(m_{l}-1\right) k_{0} \\
= & R_{k}+\left(m_{k}-2\right)+\left(2 m_{k}-1\right) k_{0} \\
& \quad-\left(2 m_{k}-1\right) B\left(m_{k}-1\right) k_{0} .
\end{aligned}
$$

Man setze hier $\sum_{l=k+1}^{k_{0}-1} h_{l}=m_{k+1}-m_{k_{0}}=m_{k}-2$ ein, da $h_{k}=m_{k_{0}}=1$ ist. Dabei ergibt sich die letzte Gleichheit hier oben aus Hilfssatz 1 und dem Sachverhalt $\sum_{l} h_{l} B\left(m_{l}-1\right)=\sum_{m=2}^{m_{k}} B(m-1)-B\left(m_{k}-1\right)$. Letzterer folgt aus den Fällen $h_{l}=1$ oder $h_{l}=0$. Die Abschätzung $\left|R_{k}\right| \leq m_{k}$ ist leicht mittels Hilfssatz 3 zu beweisen. Folglich finden wir

$$
k_{0}-k<\frac{2 m_{k}-2}{2 m_{k}-1}+\left\{1-B\left(m_{k}-1\right)\right\} k_{0}
$$

und eine Hälfte von (10) ist bewiesen. Allerdings ist diese Ungleichung nur dadurch erreicht, daß die Induktionsannahme in der Form,$\ldots<1$ " eingesetzt wurde. Nimmt man anstatt dessen ,...>-1", so entsteht eine untere Schranke indem man das Vorzeichen am Glied $\left(m_{k}-2\right)$ vertauscht. Mithin haben wir auch

$$
k_{0}-k>-\frac{2 m_{k}-2}{2 m_{k}-1}+\left\{1-B\left(m_{k}-1\right)\right\} k_{0}
$$

gezeigt und damit den Induktionsschritt, worauf der ganze Beweis folgt. 
Bemerkung. Die Lösung von Brun basiert auf Abschätzungen wie (9). Seine Ungleichungen lassen sogar Indizes $k$ mit $h_{k}>1 \mathrm{zu}$. Allerdings wird das Endergebnis nur $|\ldots|<a_{k}$, welches nicht scharf genug ist, um die jetzige Verfeinerung zu erzielen.

Die Anzahlfunktion. Zunächst wird die Filteridentität eingesetzt, um die gewünschten Abschätzungen von $F(n)$ zu erhalten. Es seien der Einfachheit halber $\alpha=\sqrt{\pi} / 2$ und $\beta=2 / \sqrt{\pi}$ gesetzt. Wir betrachten im Folgenden nur ganze Zahlen $n \geq 2916$.

Zuerst wird die ganze Zahl $m$ so gewählt, daß

$$
\left(\frac{n}{4}\right)^{1 / 3}-\frac{7}{6}<m<\left(\frac{n}{4}\right)^{1 / 3}-\frac{1}{6}
$$

ist. Insbesondere ist $m \geq 8$ und Hilfssatz 1 gibt

$$
B(m-1)=\frac{\alpha}{\sqrt{m}}+\mathcal{O}\left(m^{-3 / 2}\right) .
$$

Nach Satz 4 ist $m \leq m_{k^{*}+1}$ und somit gibt es einen Index $k$ mit $h_{k}=1$ und $m_{k}=m$.

Wir wollen jetzt möglichst enge untere und obere Schranken von $F(n)=$ $N_{k_{0}}=k_{0}-\varepsilon, \varepsilon=0$ oder 1 , finden. Die ganze Zahlen $m, k_{0}$ und $k$ sind allesamt von $n$ abhängig. Aus Satz 6 und der Beziehung (12) geht hervor, daß

$$
k=\alpha k_{0} m^{-1 / 2}+\mathcal{O}\left(k_{0} m^{-3 / 2}\right) .
$$

Nach Hilfssatz 2 ist $k_{0}=\mathcal{O}\left(n^{1 / 2}\right)$ und dank (11) ergibt sich

$$
m k(k-1)=\alpha^{2} k_{0}^{2}+\mathcal{O}\left(n^{2 / 3}\right) \quad \text { bzw. } \quad k(k-1)=\mathcal{O}\left(n^{1 / 3}\right) .
$$

Greift man jetzt auf Hilfssatz 3 zurück, erhält man

$$
n=m k(k-1)+\mathcal{O}(k(k-1))=\alpha^{2} k_{0}^{2}+\mathcal{O}\left(n^{2 / 3}\right) .
$$

Als letzter Schritt bleibt nur noch

$$
k_{0}-\beta \sqrt{n}=\frac{k_{0}^{2}-\beta^{2} n}{k_{0}+\beta \sqrt{n}}=\mathcal{O}\left(n^{1 / 6}\right),
$$

wobei $k_{0}+\beta \sqrt{n} \geq(1+\beta) \sqrt{n}-\frac{1}{2}$ eingesetzt wurde. Somit haben wir gezeigt

SATz 7. Die Anzahl $F(n)$ der ganzen Zahlen kleiner gleich $n$ nach dem Flaviusschen Siebverfahren genügt

$$
F(n)=\frac{2}{\sqrt{\pi}} \sqrt{n}+\mathcal{O}\left(n^{1 / 6}\right) .
$$

Bemerkung. Eine Berücksichtigung der verschiedenen Ordoausdrücke und bessere Angaben in Hilfssatz 1(2) läßt die Verschärfung zu:

$$
F(n)=\frac{2}{\sqrt{\pi}} \sqrt{n}+\theta(n) n^{1 / 6}, \quad|\theta(n)|<2,6, n>2915 .
$$

Die nötige Rechnung wurde ursprünglich in [2] durchgeführt. 


\section{Literaturverzeichnis}

[1] Lösungen zu den Aufgaben 107, 116, Nord. Mat. Tidskr. 5 (1957), 114-116, 160-161 und 203-205.

[2] M. E. Andersson, Flavius såll, U.U.D.M. Project Report 1988:P4 (schwedisch).

[3] V. Brun, Un procédé qui ressemble au crible d'Eratosthène, An. Ştiinţ. Univ. "Al. I. Cuza" Iaşi Secţ. I a Mat. (N.S.) 11B (1965), 47-53.

[4] L. Carlitz, Lösung zu Aufgabe 115, Nord. Mat. Tidskr. 5 (1957), 159-160.

[5] V. Gardiner, R. Lazarus, N. Metropolis, and S. Ulam, On certain integers defined by sieves, Math. Mag. 2 (1965), 117-122.

Matematiska institutionen

Stockholms Universitet

S-106 91 Stockholm, Sverige

E-mail: mats.andersson@matematik.su.se

Eingegangen am 26.9.1996

und in revidierter Form am 12.2.1998 